\title{
OCORRÊNCIA DOS CAMARÕES Macrobrachium amazonicum (HELLER, 1862) E Macrobrachium jelskii (MIERS, 1877)
}

\author{
Welington Soares Feitosa ${ }^{1}$; Mariane Fernandes Gomes Nery ${ }^{2}$; Isis Campos de Lucena ${ }^{3}$; \\ Francisca Gesuina Sousa Landim ${ }^{4}$; Allysson Pontes Pinheiro ${ }^{5}$
}

\begin{abstract}
Resumo
Os camarões Macrobrachium amazonicum e Macrobrachium jelskii são espécies essencialmente dulcícolas, distribuídos nas regiões tropicais e subtropicais da América do Sul. O objetivo do estudo foi registrar a presença dessas espécies no Rio Cariús na bacia do Alto Jaguaribe, no município de Farias Brito, na região semiárida do sul no estado do Ceará, Brasil. As coletas foram realizadas de junho de 2013 a janeiro de 2014. Os espécimes foram transportados e depositados na coleção carcinológica (0004 e 0005) do Laboratório de Crustáceos do Semiárido da Universidade Regional do Cariri e na Coleção Carcinológica do Museu de Zoologia da USP (MZUSP 32647 e MZUSP 32646).
\end{abstract}

Palavras-chave: Decapoda. Dulcícola. Nordeste.

\section{OCCURRENCE OF THE PRAWNS Macrobrachium amazonicum (HELLER, 1862)}

AND Macrobrachium jelskii (MIERS, 1877)

\begin{abstract}
Macrobrachium amazonicum and Macrobrachium jelskii are essentially freshwater species distributed in tropical and subtropical regions of South America. This paper reported the occurrence of these species in the Cariús River of the Upper Jaguaribe River Basin in the municipality of Farias Brito in the semiarid region in Ceará state, Brazil. Collections were carried out from June 2013 to January 2014. Voucher specimens were deposited in the Collection (0004 and 0005) of the Laboratory of Crustaceans of the Semiarid Region, Universidade Regional do Cariri, Brazil and at the Collection Carcinologica of Museu de Zoologia da Universidade de São Paulo MZUSP (32647 and 32646).
\end{abstract}

Keywords: Decapoda. Freshwater. Northeast.

\footnotetext{
${ }^{1}$ Professor efetivo do estado do Ceará. Autor correspondente: welingtoncariautaba@ hotmail.com.

${ }^{2}$ Supervisora do PIBID Diversidade do Procampo -URCA

${ }^{3}$ Doutoranda em Ecologia e recursos naturais da Universidade Federal do Ceará

${ }^{4}$ Professora de Escola Profissional do estado do Ceará

${ }^{5}$ Professor do Depto. de C. Biológicas da URCA
} 


\section{Introdução}

O gênero Macrobrachium (BATE, 1868) é composto por 243 espécies (DE GRAVE et al., 2009) distribuídas em regiões tropicais e subtropicais em todo o mundo (MELO, 2003). No Brasil, encontram-se 18 espécies de Macrobrachium, alguns dos quais estão sendo usados como isca em pescarias com anzol e alimentos para a população ribeirinha, além de compor uma parte importante na teia trófica de ambientes limínicos (RAMOS-PORTO; COELHO, 1998). O conhecimento da carcinofauna brasileira do semiárido é ainda pobre e difusa. No entanto, alguns trabalhos recentes devem ser destacados com camarões do gênero Macrobrachium, tais como Macrobrachium jelskii em Sergipe, Ceará e Pernambuco (CIRILO et al., 2011; LANDIM, 2014) Macrobrachium amazonicum no Ceará e Pernambuco (NERY, 2014) e caranguejos em Pernambuco. Na zona rural do nordeste do Brasil, duas espécies são generalizadas Macrobrachium amazonicum e M. jelskii.

Macrobrachium amazonicum foi descrito pela primeira vez para o rio Amazonas e foi encontrado na Venezuela, Guiana Inglesa, Suriname, Guiana francesa, Equador, Peru, Bolívia, Paraguai, Argentina e Brasil (nos Estados do Amapá, Pará, Amazonas, Maranhão, Piauí, Ceará, Rio Grande do Norte, Paraíba, Pernambuco, Acre, Mato Grosso, Bahia, Goiás, Minas Gerais, Mato Grosso do Sul e Paraná) (MELO, 2003; ALMEIDA 2008; SILVA, 2010).

Macrobrachium jelskii foi descrito pela primeira vez na região das Guianas de Oiapoque e encontra-se na Venezuela, Trinidad, Guiana Inglesa, Suriname, Guiana francesa, Bolívia, Argentina e Brasil (nos Estados do Amapá, Pará, Amazonas, Maranhão, Ceará, Rio Grande do Norte, Paraíba, Pernambuco, Mato Grosso, Bahia, Alagoas, Sergipe, Minas Gerais, Espírito Santo, São Paulo e Santa Catarina) (MELO, 2003; COLLINS, 2000; ALMEIDA, 2008; SILVA, 2010).

Este trabalho objetivou registrar a ocorrência de M. amazonicum e M. jelskii no Rio Cariús no município de Farias Brito, Ceará, Brasil.

\section{Material e Métodos}

Com o intuito de se aprofundar o conhecimento sobre a fauna de Decapoda do semiárido brasileiro, foram realizadas coletas de material biológico entre os meses de junho de 2013 a janeiro de 2014. Os espécimes foram coletados com o auxílio de armadilhas e 
peneiras distribuídas nas margens do Rio Cariús, município de Farias Brito (0649'12.1 "S e 39³1'39" W), que faz parte da Bacia do Alto Jaguaribe, no estado do Ceará. O rio Cariús tem aproximadamente 140 quilômetros, subindo para a cidade de Santana Cariri-CE e desaguando no rio Jaguaribe na cidade de Jucás-CE, apresentando vegetação marginal com árvores grandes e pequenas, variando de 4 a 6 metros de profundidade. A precipitação média no local de coleta foi de $32,8 \mathrm{~mm}$, de acordo com a Fundação Cearense de Meteorologia e Recursos Hídricos (FUNCEME, 2014).

\section{Resultados e Discussão}

Foram coletados 19 indivíduos de Macrobrachium amazonicum (Fig. 1) e 2854 de Macrobrachium jelskii (Fig. 2) (Tabela 01). Tais espécimes foram tombados e depositados na

coleção carcinológica do Laboratório de Crustáceos do Semiárido (LACRUSE) sob a numeração (0004 e 0005) e na coleção do Museu de Zoologia da Universidade de São Paulo (32646 e 32647) (M. amazonicum e M. jelskii respectivamente).

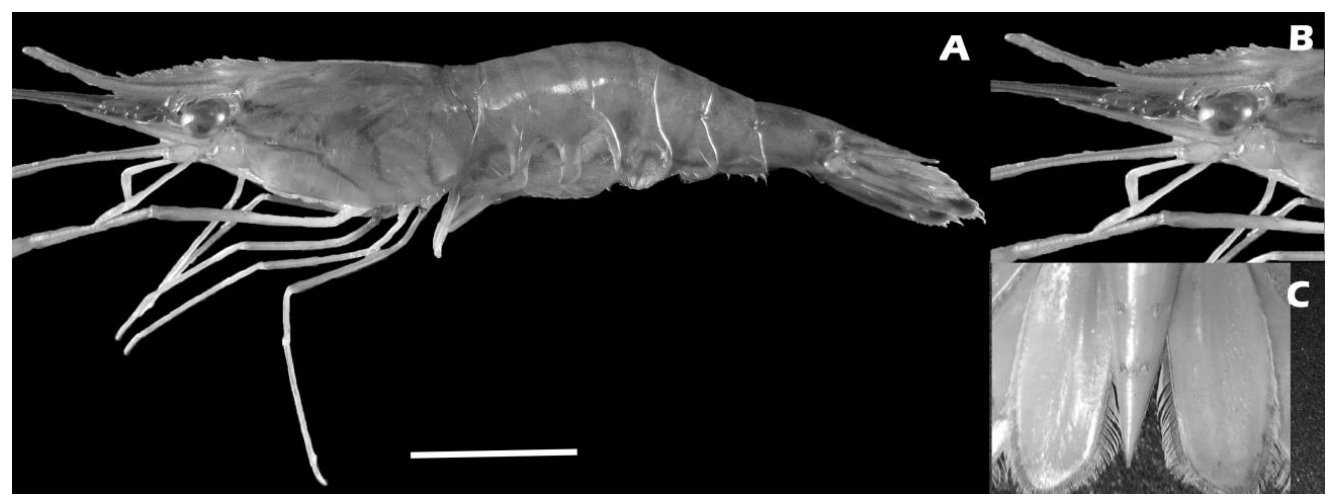

Figura 1. Macrobrachium amazonicum (Heller, 1862), vista lateral. Escala: $1 \mathrm{~cm}, \mathrm{~B}$ : Detalhe do Rostro, C: Detalhe do Telson 


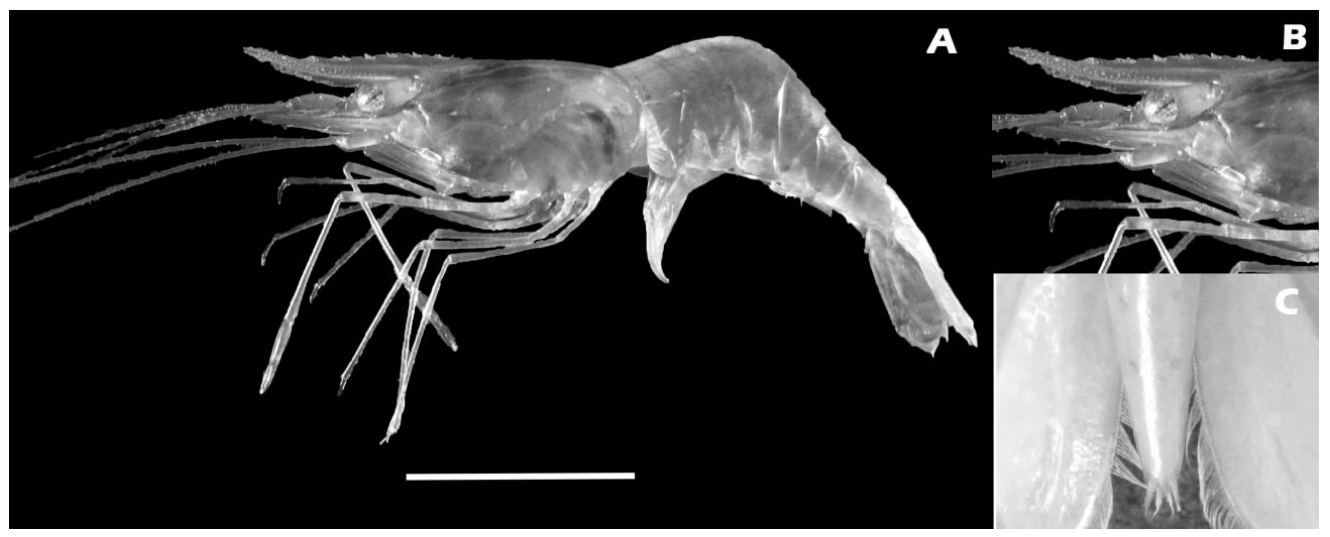

Figura 2. Macrobrachium jelskii, vista lateral. Escala: $1 \mathrm{~cm}, \mathrm{~B}$ : Detalhe do Rostro, C: Detalhe do Telson

Tabela 1: Frequência dos espécimes coletados entre os meses de junho de 2013 a janeiro de 2014

\begin{tabular}{l|c|c}
\hline \multicolumn{1}{c|}{ Mês } & M. amazonicum & M. jelskii \\
\hline Junho 2013 & 0 & 600 \\
\hline Julho 2013 & 0 & 416 \\
\hline Agosto 2013 & 4 & 568 \\
\hline Setembro 2013 & 9 & 369 \\
\hline Outubro 2013 & 1 & 293 \\
\hline Novembro 2013 & 1 & 238 \\
\hline Dezembro 2013 & 3 & 198 \\
\hline Janeiro 2014 & 1 & 2854 \\
\hline \multicolumn{1}{c|}{ Total } & 19 & \\
\hline
\end{tabular}

Apesar de ambas as espécies possuírem registros anteriores para o Estado do Ceará, este é o primeiro registro desses camarões para a Bacia do Alto Jaguaribe (Fig.3). Tal constatação pode residir no fato de que a carcinofauna de regiões continentais brasileiras tem sido alvo de estudos escassos e descontínuos (BIALETZKI et al., 1997; FREITA et al., 2013; MELO, 2003). Tal situação é preocupante pela grande pressão que o ambiente tem sofrido devido às atividades antrópicas. Desta forma, pode-se estar comprometendo a biodiversidade de uma região antes mesmo dela ser conhecida. 


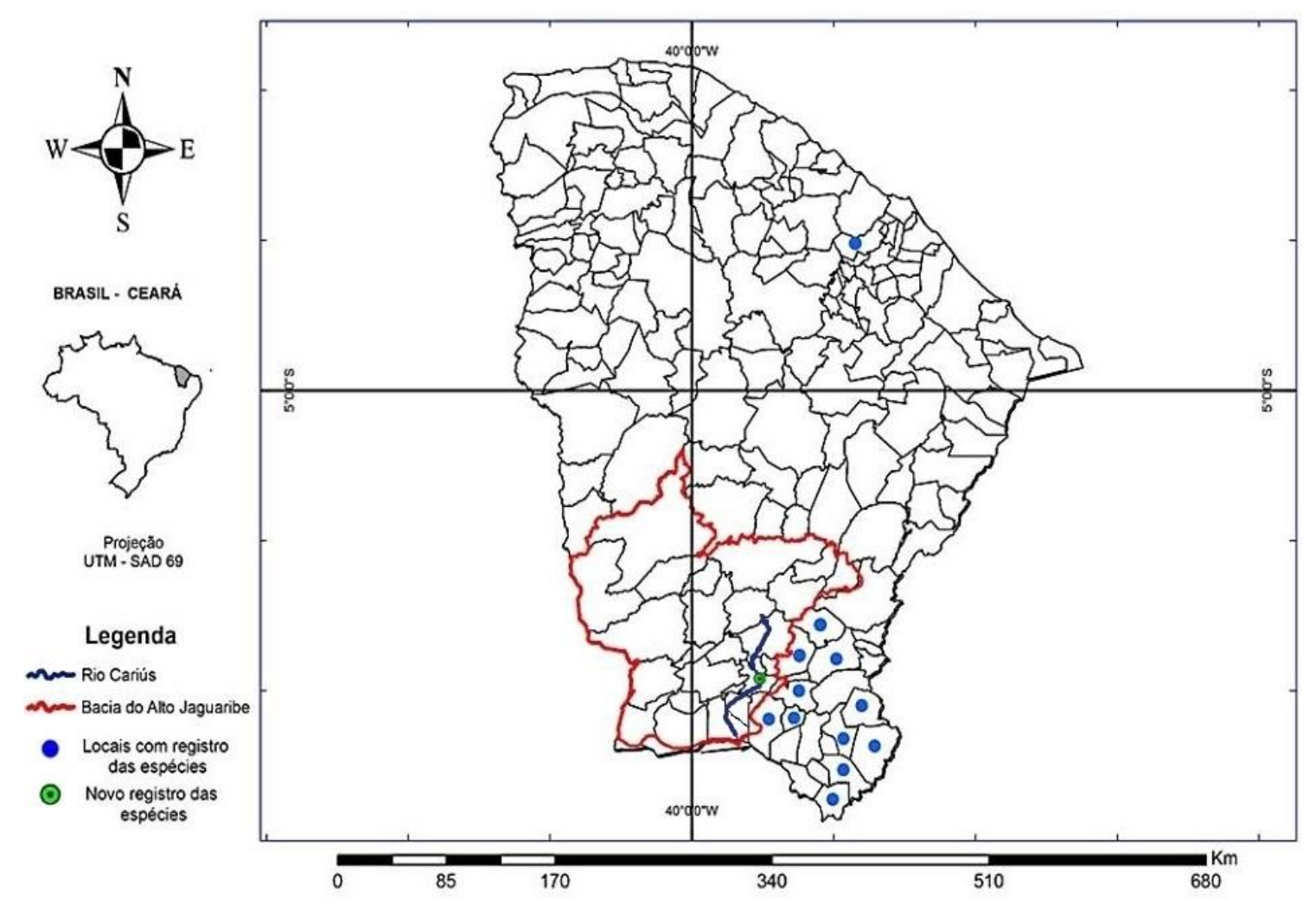

Figura 3: Ocorrência de Macrobrachium amazonicum e Macrobrachium jelskii no estado do Ceará, Brasil

O Rio Cariús é um rio intermitente sazonal, que se caracteriza por apresentar vazão positiva durante os períodos úmidos tornando-se nula nas épocas de estiagem. Seu leito tem feições retilíneas, pois grande parte do seu trajeto é controlado por uma linha de falha tectônica (PRIMO, 2008).

A ocorrência das duas espécies na região em questão, não é fato surpreendente, mas reafirma a necessidade de compreender melhor como espécies exclusivamente aquáticas lidam com os longos períodos de seca característicos do semiárido nordestino do Brasil. Além disso, o Departamento Nacional de Obras Contra as Secas (DNOCS), órgão público brasileiro que lida com o fenômeno da seca, aprovou nesses reservatórios a construção de barragens, como uma política de gestão; introdução de espécies de peixes e crustáceos (DNOCS, 2014). Tais espécies não necessariamente são naturais dos locais de liberação. Essas iniciativas não possuem qualquer grau de acompanhamento de seu impacto nas populações naturais e precisam ser avaliadas com maior atenção, para evitar problemas relacionados à introdução de espécies exóticas nesses ecossistemas. Apesar do exposto não está claro se o presente registro 
de ambas espécies trata-se do resultado dessas introduções, ou se as mesmas são naturais da região em questão.

\section{Conclusão}

O presente trabalho encontrou novos locais de ocorrência para $M$. amazonicum e $M$. jelskii na Bacia hidrográfica do Alto Jaguaribe, região semiárida do Ceará, no nordeste brasileiro, servindo como base para estudos posteriores.

\section{Referências}

ALMEIDA, A. O.; COELHO P. A.; FERRAZ N.R.; LUZ J. R.; SANTOS J. T. A. Decapod crustaceans in fresh Waters of southeastern Bahia, Brazil. Revista de Biologia Tropical, v.56, p. 1225-1254. 2008.

BATE, C.S. On a new genus, with four new species of freshwater prawns. Proceedings of the Royal Society of London, p. $363-368,1868$.

BIALETZKI, A; BAUMGARTENER, G; BOND-BUCKUP, G.;NAKATANI, K. Occurrence of Macrobrachium amazonicum (Heller, 1862) (Decapoda, Palaemonidae) in Leopoldo's Inlet (Ressaco do Leopoldo), upper Paraná river, Porto Rico, Paraná, Brasil. Revista Brasileira de Zoologia, v.14, n. 2, p. 379-390, 1997.

CIRILO, A.T.O.; NUNES M.L.;SANTOS M.C. Caracterização física e nutricional do camarão "saburica" (Macrobrachium jelskii, Miers, 1877) e de produtos derivados. Scientia Plena, v.7, n.7.p. 1-7, 2011.

COLLINS, P.A. A new distribution record for Macrobrachium jelskii (Miers, 1877) in Argentina (decapoda, palaemonidae). Crustaceana, v.73, n. 9, p.1167-1169, 2000. 
DE GRAVE, S.; AHYONG, S.T.; CHAN, T.Y.; CRANDALL, K.A.; DWORSCHAK, P.C. A.;PENTCHEFF, N. D. Classification of living and fossil general of decapod crustaceans. The Raffles Bulletin of Zoology, v.21, p.1-109, 2009.

DNOCS. DEPARTAMENTO DE OBRAS CONTRA AS SECAS. Disponível em: 〈http://www.dnocs.gov.br/〉. Acesso em: 23 out. 2014.

FREITA, F.R.V.; SANTANA N.N.; LANDIM F.G.S.; PEIXOTO B.M.E.; PINHEIRO A.P. Occurrence of Goyazana castelnaui (H. Milne-Edwards, 1853) (Crustacea: Decapoda: Trichodactylidae) in the semi-arid region of the state of Pernambuco, Brazil. Pan-American Journal of Aquatic Sciences, v. 8, n.4, p.358-360, 2013.

FUNCEME. FUNDAÇÃO CEARENSE DE METEOROLOGIA E RECURSOS HÍDRICOS. Disponível em: 〈http://www.funceme.br/〉. Acesso em: 21 set. 2014.

HELLER, C. Beiträge zur näheren Kenntniss der Macrouren. S.B Akad. Wien. v.45, p. 389-426, 1862.

LANDIM, F.G.S.O. Variação morfológica do cefalotórax de Macrobrachium jelskii (Miers, 1778) (Crustacea, Decapoda, Palaemonidae), mediante aplicação da morfometria geométrica. 2014. 72 f. (Mestrado em Bioprospecção Molecular) Universidade Regional do Cariri, Crato, CE, 2014.

MELO, G.A.S. Manual de Identificação dos Crustacea Decapoda de água doce do Brasil. São Paulo: Centro Universitário São Camilo, Museu de Zoologia, Universidade de São Paulo, Loyola, 2003.

MIERS, E. J. On a collection of Crustacea, Decapoda and Isopoda, chiefly from South America, with descriptions of new general and species. Proceedings of the Zoological Society of London, p. 653-679, 1877.

NERY, M.F.G. Morfometria Geométrica do camarão-da-amazônia Macrobrachium amazonicum (Heller, 1862) (Crustacea, Decapoda, Palaemonidae) em duas bacias 
hidrográficas do nordeste. 2014. 80 f. (Mestrado em Bioprospecção Molecular) Universidade Regional do Cariri, Crato, CE, 2014.

PRIMO, E. A. Impactos ambientais no rio Cariús no distrito de Cariutaba, Farias BritoCE. 2008. 60 f. (Especialização em Meio Ambiente) - Universidade Regional do Cariri, Crato, CE. 2008.

RAMOS-PORTO, M.; P.A. COELHO. Malacostraca-Eucarida-Caridea (Alpheoidea excluded); in/: YOUNG, P. S. Catalogue of Crustacea of Brazil. Rio de Janeiro, Museu Nacional, p.325-350,1998.

SILVA, J. C. Biologia e ecologia dos camarões de água doce Macrobrachium amazonicum (Heller 1862) e Macrobrachium jelskii (Miers 1778) (crustacea: caridea: palaemonoidea) no Rio Grande, região de Planura, MG. 2010. 101 f. (Tese de doutorado) - Universidade Estadual Paulista, São Paulo, 2010.

Recebido: 30/04/2015

Aceito: 28/06/2016 